\title{
ON THE MEASUREMENT OF IMAGE DISTURBANCE IN AERIAL PHOTOGRAPHY
}

\author{
H.J. Tiziani \\ Institute of Applied Optics \\ University Stuttgart, Pfaffenwaldring 9, 7000 Stuttgart 80
}

\section{Introduction}

Image quality together with the correction of distortion is very important for airborne photographs used in photogrammetry. Progress in the optical design as well as in production technology and film resolution improved the storage capacity of information to $5 \times 10^{8}$ pixels in the image. Distortion can be corrected to be in the region of one micrometer eventhough it needs only to be known in order to be corrected using image processing.

Flight conditions usually cause disturbance of image quality. The optical transfer function (OTF) and especially the modulation transfer function (MTF) are useful techniques for measuring the image quality of photographs. The MTF of the image forming system is the result of the contributions of the links in the image transfer forming chain, namely, the camera, the film, image motion (in systems without forward motion compensation, FMC), vibrations, and the atmosphere. ${ }^{1-5}$

For the analysis of the image forming systems, artificial or natural edges are imaged the edge gradient analysis (EGA), and varying spatial frequency patterns are used for the grating pattern technique. The aim of this paper is to analyse the influence of image motion, vibration and the atmosphere on the image quality. Results are shown and discussed. 
Edge spectrum ratio for determining the MTF from an edge image

During the past 20 years different methods for edge gradient analysis, EGA, have been developed. The differentiation of the edge, has often been used to obtain the modulation transfer function. The optical Transfer Function (OTF) respectively the MTF can be determined by the Fourier Transform of the spread function, obtained by differentiating the edge image. The differential method works well for noise free edge analysis. Recently, a more direct method using a Hanning window was developed ${ }^{3}$. The new method is based on the Fourier spectrum amplitude ratio of the real and an ideal edge image. It can be called an edge spectrum ratio method; where the MTF is obtained by dividing the spatial frequency spectrum of a scanned edge, imaged by the system to be analysed, by the spatial frequency spectrum of an ideal edge. However the edge to be scanned is of limited extend. Using a window as a rectangular function leads to zero amplitudes at even harmonic frequencies in the spectrum of the ideal edge. To avoid a division by zero, the rectangular function needs to be replaced by a more suitable window function such as the Hanning function.

Figure 1 shows schematically the procedure for the MTF determination. Before applying the Fourier transformation, both scanned and ideal edges must be multiplied by the Hanning function. By the introduction of the Hanning function, noise on top of an edge image can also be suppressed. Furthermore a convolution of the MTF with a chosen Gauss function can be used to suppress photographic grain noise.

It can be shown that a multiplication with a Hanning function does not affect the $\mathrm{MTF}^{3}$. The method was also analyzed with a computer simulated edge function and good agreement with the expected MTF was found by simulation. The ratio method requires little numerical effort in comparison to other edge detection methods. 
MTF's obtained from an edge image

To analyse the image quality of airborne photographs, artificial patterns were produced and photographed as well as natural objects containing edges. To obtain the edge image scan, a computer controlled microdensitometer was used. From the edge image the Fourier Transform was computed after the multiplication with the Hanning function. The ratio of the Fourier transformed edge images from the real and reference edges leads to the system MTF.

Fig. 2 shows the MTFs obtained from artificial and natural edges (a) in flight direction and (b) perpendicular to the flight direction. It should be noted that the difference of the MTF's of a natural and an artificial edge is small. For comparison, artificial grating structures were placed on a test field and then photographed and scanned with a microdensitometer and analysed. The solide lines of the MTF curves in Fig. 2 are the results of a scanned grating pattern. In addition agreement of the results obtained with different test patterns were obtained. For an additional test, three-bar patterns with different spatial frequencies were examined. They were put close to the grating and edge patterns and were analysed to determine resolution limits. With a $300-\mathrm{mm}$ focal length lens, the minimum detectable period in the flight direction was $100 \mathrm{~mm}$, and the corresponding spatial frequency on the film was $31,5 \mathrm{~mm}^{-1}$. The minimum detectable period perpendicular to the flight direction was $70,7 \mathrm{~mm}$ with a corresponding spatial frequency on the film of $44,6 \mathrm{~mm}^{-1}$. By introduction of a threshold in Figure 2, the resolution mentioned above is obtained by the intersection between the MTF and the threshold ${ }^{1}$. The spatial frequencies at the intersection of the MTF, for well-defined artificial edges, and the threshold curves compare well with the resolution limits obtained from the three-bar pattern in the flight direction and perpendicular to it, respectively. 


\section{Image quality degradation by linear image motion}

There are different types of image motions such as linear, parabolic, sinusoidal, and random ${ }^{2}$. This paper deals with linear motion. The quality of airborne photographs can be improved by applying forward motion compensation (FMC). The photographs thus far refered to in this paper were obtained without using FMC. It is therefore not surprising that the MTF obtained from test patterns oriented in the flight direction is worse than perpendicular to it. From the difference of the MTFs in the two directions, the image motion can be obtained. It will be compared with the theoretical image motion.

The image displacement during exposure time $\mathrm{T}$ is

$$
\mathrm{a}=\mathrm{vTf} / \mathrm{H}
$$

where $v$ is the speed of the airplane, $f$ is the focal length of the objective, and $\mathrm{H}$ is the altitude of the airplane. The spread function corresponding to the movement is a rectangular function of width a. The corresponding MTF is its Fourier transform

$$
\mathrm{MTF}_{\mathrm{B}}=a \frac{\sin \pi \mathrm{Ra}}{\pi_{\mathrm{Ra}}}
$$

where $R$ is the spatial frequency and $a$ is the image motion. If the MTF is measured perpendicular to the flight direction and multiplied by the sinc-function, the result measured in flight direction is obtained providing the signal-to-noise ratio is appropriate. According to linear response theory, the MTF in flight direction can be written as

$$
\mathrm{MTF}_{\mathrm{F}}=\mathrm{MTF}_{\mathrm{B}} \cdot \mathrm{MTF}_{\mathrm{P}}
$$

where $\mathrm{MTF}_{\mathrm{P}}$ is perpendicular to the flight direction and $\mathrm{MTF}_{\mathrm{B}}$ is in the 
direction of linear image motion. The image motion was therefore extracted from the measured $\mathrm{MTF}_{\mathrm{F}}$. For the flight parameters:

$$
v / H=0.043421 / \mathrm{sec}, T=1 / 700 \mathrm{sec}, f=303.64 \mathrm{~mm}
$$

and from Equation 4 the image motion is $a=19 \mu \mathrm{m}$.

Figure 3 shows the MTF of image motion with $a=19 \mu \mathrm{m}$. In figure $3 \mathrm{~b}$ ) $\mathrm{MTF}_{\mathrm{F}}, \mathrm{MTF}_{\mathrm{P}}$, and the product of $\mathrm{MTF}_{\mathrm{B}}$ and $\mathrm{MTF}_{\mathrm{P}}$ are shown together. Good agreement was obtained between $\operatorname{MTF}_{F}$ and $\operatorname{MTF}_{\mathrm{B}} \times \mathrm{MTF}_{\mathrm{P}}$.

\section{Image disturbance by the atmospheric influence}

In aerial cameras without forward motion compensation system (FMC), the image motion is a dominant element to reduce image quality of airborne photographs, 2,4 therefore vibrations and the atmospheric disturbance were very often neglected. After the compensation of image motion with FMC, image disturbance by vibration and the atmosphere become increasingly important. The atmospheric influence includes, among other things, turbulence, contrast degradation due to atmospheric background, and forward scattering by airborne particles ${ }^{3-5}$, etc. The atmospheric influences are based on molecular absorption of gas dissolved in air, on scattering of aerosol and dust particles, and on local short and long time changes of the refractive index. Beside the visible disturbances, the meteorological parameters are of great importance, for example, atmospheric pressure, humidity, temperature, temperature gradient and wind speed. These parameters vary with time as well as spatially. The investigations of the atmospheric influence on image quality are complicated, statistical methods need to be used. The atmospheric influences were however studied in optical astronomy theoretically, and verified by practical measurements. The influence of the atmosphere on airborne photographs was rarely considered and very few reports on investigations were 
found. 3-5 Furthermore, in astronomical photography the wavefront distortion by atmospheric disturbance can be compensated by adaptiv optics.

Some aspects on atmospheric disturbance in airborne photograph will be discussed only. There are different models to simulate the influence of the atmosphere. In addition, different atmospheric phenomena need to be considered in aerial photography:

1. atmospheric turbulence;

2. contrast degradation due to atmospheric background; ${ }^{3-5}$

3. forward scattering by airborne particles; ${ }^{5}$

4. aircraft boundary layer turbulence.

The influence of each phenomenon can be described by an MTF. Fig. 4 shows schematically the image transfer chain linked by the different atmospheric MTFs.

The atmospheric turbulence, for instance arises from permanent mixture of cold and warm layers of air leading to local density and humidity variations. The density and humidity variations act as temporal and spatial refractive index variations. Intensity variations due to turbulence can be represented approximately by a log-normal distribution. The MTF of atmospheric turbulence has been derived from the log-normal model, using the Fried parameter. The atmospheric turbulence can be approximated by using a single parameter $r_{0}$, which was defined by Fried ${ }^{7}$.

\section{Measurement results at different flight altitudes}

For the experimental verification of some theoretical models, two artificial edge and grating patterns were laid out on the ground of a test field perpendicular to each other. To examine the atmosphere, different airborne photographs were taken by Leica Heerbrugg 
corporation from three different flight altitudes. The weather condition and camera parameters are summarized in table 1 . While exposing, there was a relatively strong haze with a clear top limit at about $1800 \mathrm{~m}$ above sea level. As shown in the table 1, for instance, three airborne photographs have been taken shortly after each other, so that the same atmospheric conditions could be assumed. The resulting MTFs at different flight altitudes were determined not only with artificial edge patterns but also with grating patterns. Fig. 5 shows only the MTF curves measured from edge images. The same results were obtained with grating patterns.

In Fig. 5, two MTF curves for each flight altitude are shown together. The curves in $\mathrm{x}$-direction correspond to the patterns in flight direction, and the Y-direction is perpendicular to it. We found good agreement of the MTF curves obtained from both edge patterns. on account of the larger transfer path, the MTF at the flight altitude of $3435 \mathrm{~m}$ above sea level is worse than the one at $1920 \mathrm{~m}$ above sea level. According to the theories, the MTF considering different atmospheric influences decreases with altitude. But unexpected, the curves for a flight altitude of $900 \mathrm{~m}$ above sea level lie lower than those for $1920 \mathrm{~m}$ above sea level. This is probably due to the relative strong haze below $1800 \mathrm{~m}$ above sea level. At $900 \mathrm{~m}$, the haze was close to the entrance pupil of the camera leading to a considerable influence on the image quality. Because the nearer the turbulent layer to the camera entrance pupil, the greater the influence on image quality is expected. Furthermore, it is possible that the aircraft has created stronger boundary layer turbulence due to the strong haze. Moreover, the visibility from the aircraft was worst at $900 \mathrm{~m}$ (see table 1). For example, the inclined sight visibility was $12-18 \mathrm{~km}$. For imaging an object on the ground, the inclined sight visibility is essential. The bad visibility reduces the contrast of the object and the image. At $1920 \mathrm{~m}$ and $3435 \mathrm{~m}$ the inclined sight visibility were approximately equal. The atmospheric influence on the MTF's for the last two heights agrees with those theoretically expected. 
It should be noted that by knowing the MTF curves of the camera lens and the storage medium such as the film an MTF of the disturbance like image motion, vibration or atmospheric influence can experimentally be determined by dividing the measured MTF by the known MTFs. It can, in addition, be compared with the theoretical models. The models to calculate the MTF of the atmosphere can be verified by measuring the resulting MTF's at different flight altitudes.

It was found experimentally that the predicted influence of the atmospheric turbulence according to Fried's model was smaller than obtained by MTF measurements. An explanation could be that additional atmospheric influences, such as those mentioned in Fig. 4, need to be taken into consideration. It is unlikely that mechanical influences from the camera carrier were different for different photographs. However the aircraft boundary layer turbulence might be different for different photographs. According to the theory, the contrast degradation due to atmospheric background, and the forward scattering by airborne particles are dependent on the altitude, where the former is independent and the latter quasi independent on the spatial frequency. Both influences appear to increase with increasing spatial frequencies according to the measurments. Therefore, it is necessary to enlarge and modify the models.

\section{Conclusions}

The MTF of the image forming system was analysed. The influence of image motions, vibrations as well as the atmosphere can be examined. The measurements show that image motion and the atmosphere can be a limiting link in the image forming chain. By image motion compensation the performance of the image forming system can very much be improved. In addition the MTF's of the lens systems were improved in the last years. However vibrations and the atmosphere can lead to reduction on the improved image quality. According to the models, the atmospheric influences increase with flight altitude, but haze close to the 
entrance pupil of the camera can reduce image quality drastically. This was verified by some measurements. The atmospheric influences on image quality should be examined further.

\section{Acknowledgment}

The contributions by Dr. Fang Lei and the Leica Heerbrugg (Wild) corporation for the submission of the airborne photographs are gratefully acknowledged.

\section{References}

1. Tiziani, H.J.,

The use of optical Transfer Function for Assessing the Quality of optical Systems: Photogrammetria, 34, pp.45-68, (1978).

2. H.J. Tiziani

Beurteilung der Bildqualität von Luftbildkammern:

Vermessung, Photogrammetrie, Kulturtechnik. 4-77, pp.137-146, (1977)

3. Fang Lei and H.J. Tiziani,

A comparison of Methods to Measure the Modulation Transfer Function of Aerial Survey Lens systems from the Image Structures, Photogrammetric Engineering and Remote Sensing, $54(1), 41-46(1988)$.

4. H. -K. Meier,

Progress by Forward Motion Compensation for zeiss Aerial Cameras, Bildmessung und Luftbildwesen, 52, 143-152 (1984).

5. D. Wulich and N.S.Kopeika, Image resolution limits resulting from mechanical vibrations, Opt. Eng. 26, 529-533(1987).

6. N.S.Kopeika, Imaging through the atmosphere for airborne reconnaissance, Opt.Eng.26(11), 1146-1154 (1987).

7. D.L.Fried, Statistics of a Geometric Representation of Wavefront Distortion, J.opt.Soc.Am.55(11), 1427-1435(1965).

8. F.Lei, H.J.Tiziani, Atmospheric influence on image quality of airborne photographs, optical Engineering (in print). 


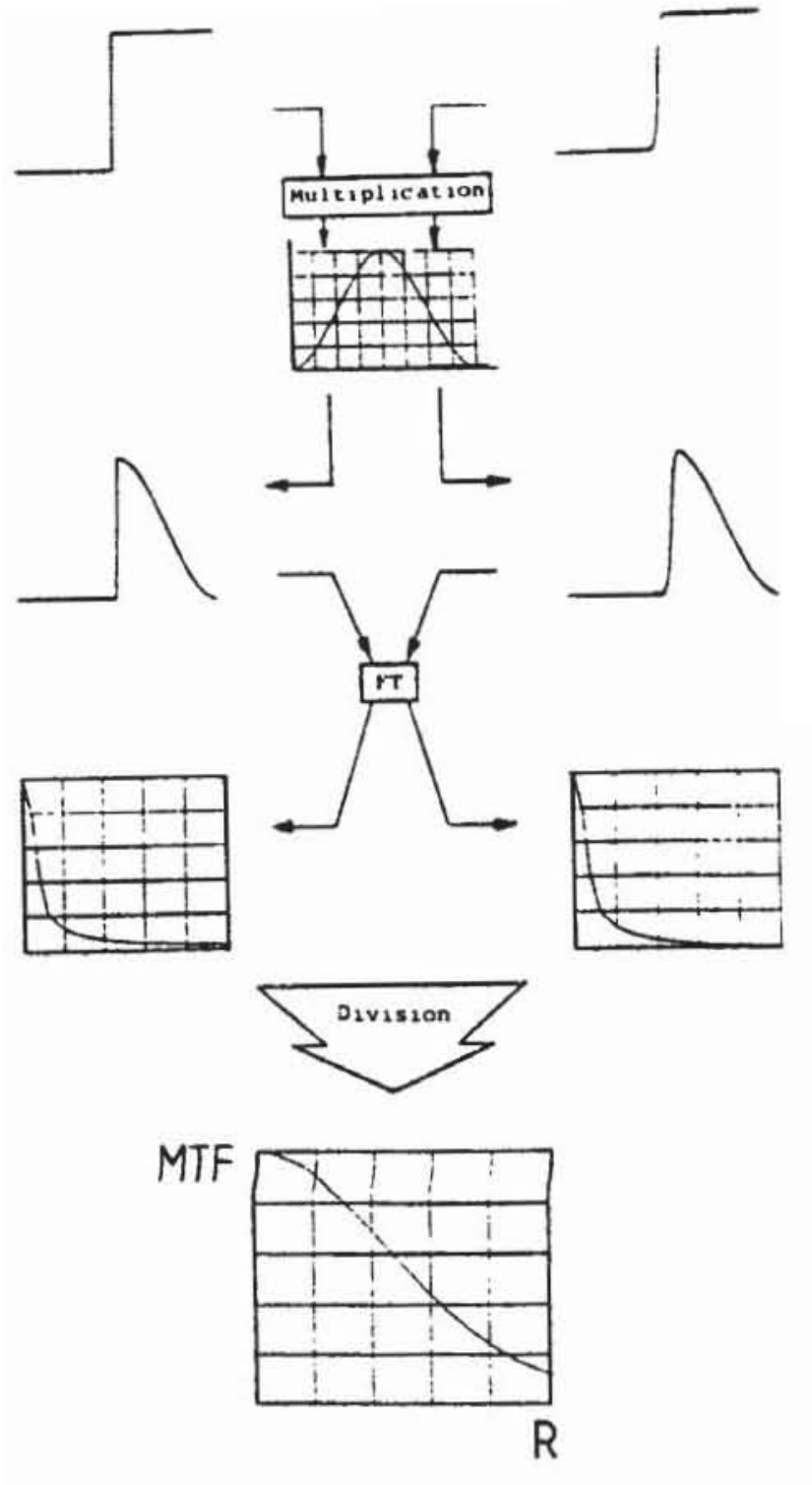

Fig. 1:

Diagram to calculate the MTF from edge scans using the Hanning function
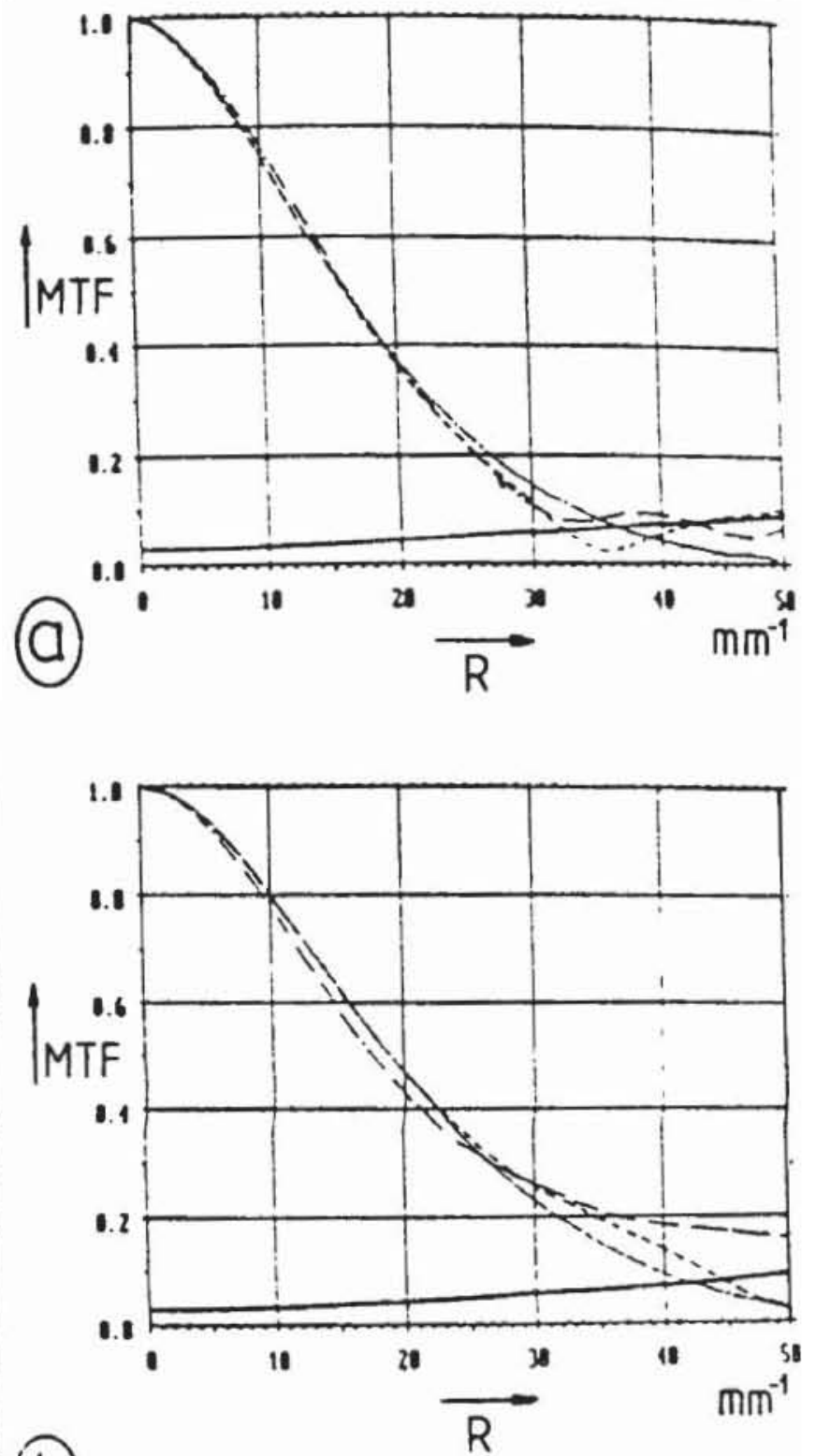

(b)

Fig.2:

a) MTF of the test patterns in flight direction

b) MTF of the test patterns perpendicular to the flight direction MTF curve of a grating pattern MTF curve of an artificial edge image MTF curve of a natural edge image Threshold value SW 

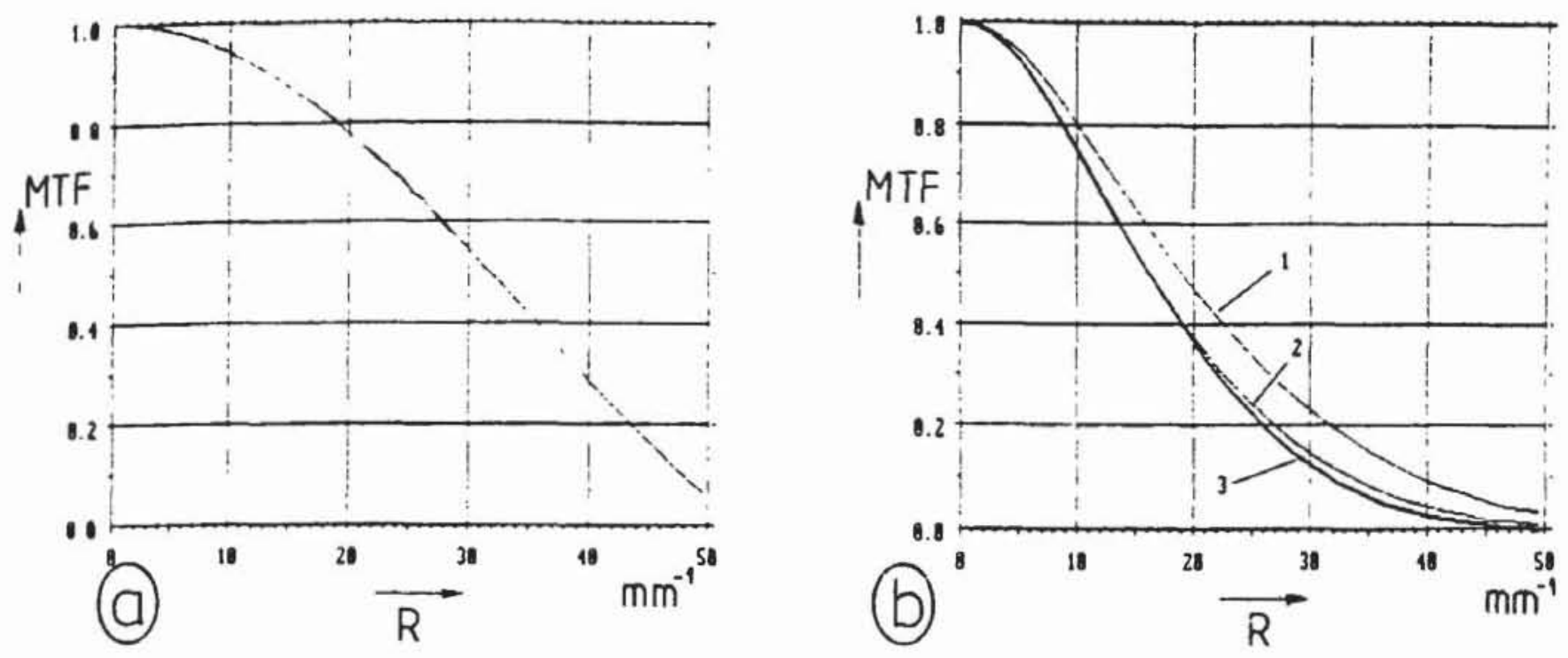

Fig. 3 :

a) MTF of an image motion with $a=19 \mu \mathrm{m}$

b) $1: \mathrm{MTF}_{\mathrm{P}} \quad 2: \mathrm{MTF}_{\mathrm{F}} \quad 3: \mathrm{MTF}_{\mathrm{B}} \times \mathrm{MTF}_{\mathrm{P}}$

\begin{tabular}{|c|c|c|c|c|}
\hline \multirow[t]{2}{*}{ No. } & \multirow{2}{*}{$\begin{array}{l}\text { time } \\
\text { (h) }\end{array}$} & \multirow{2}{*}{$\begin{array}{l}\text { flight altitude } \\
\text { above sea level }\end{array}$} & \multicolumn{2}{|c|}{ visibility from the aircraft } \\
\hline & & & inclined sight & horizontal sight \\
\hline 1 & 12.06 & $900 \mathrm{~m}$ & $12-18 \mathrm{~km}$ & $27 \mathrm{~km}$ \\
\hline 2 & 12.16 & $1920 \mathrm{~m}$ & $30-45 \mathrm{~km}$ & $40-50 \mathrm{~km}$ \\
\hline 3 & 12.35 & $3435 \mathrm{~m}$ & $30-50 \mathrm{~km}$ & $90-150 \mathrm{~km}$ \\
\hline \multicolumn{5}{|c|}{ Measurements on the ground near the test patterns } \\
\hline No. & $\begin{array}{l}\text { tempe- } \\
\text { rature }\end{array}$ & $\begin{array}{l}\text { relative } \\
\text { humidity }\end{array}$ & $\begin{array}{l}\text { air pressure } \\
(\mathrm{mb})\end{array}$ & $\begin{array}{l}\text { horizontal } \\
\text { illuminance }\end{array}$ \\
\hline 1 & $22 \circ \mathrm{C}$ & $58 \%$ & 977 & 72 '000 Lux \\
\hline 2 & $22.5^{\circ} \mathrm{C}$ & $57 \%$ & 977 & $73^{\prime} 000$ Lux \\
\hline 3 & $23^{\circ} \mathrm{C}$ & $54 \%$ & 977 & $76^{\prime} 000$ Lux \\
\hline
\end{tabular}




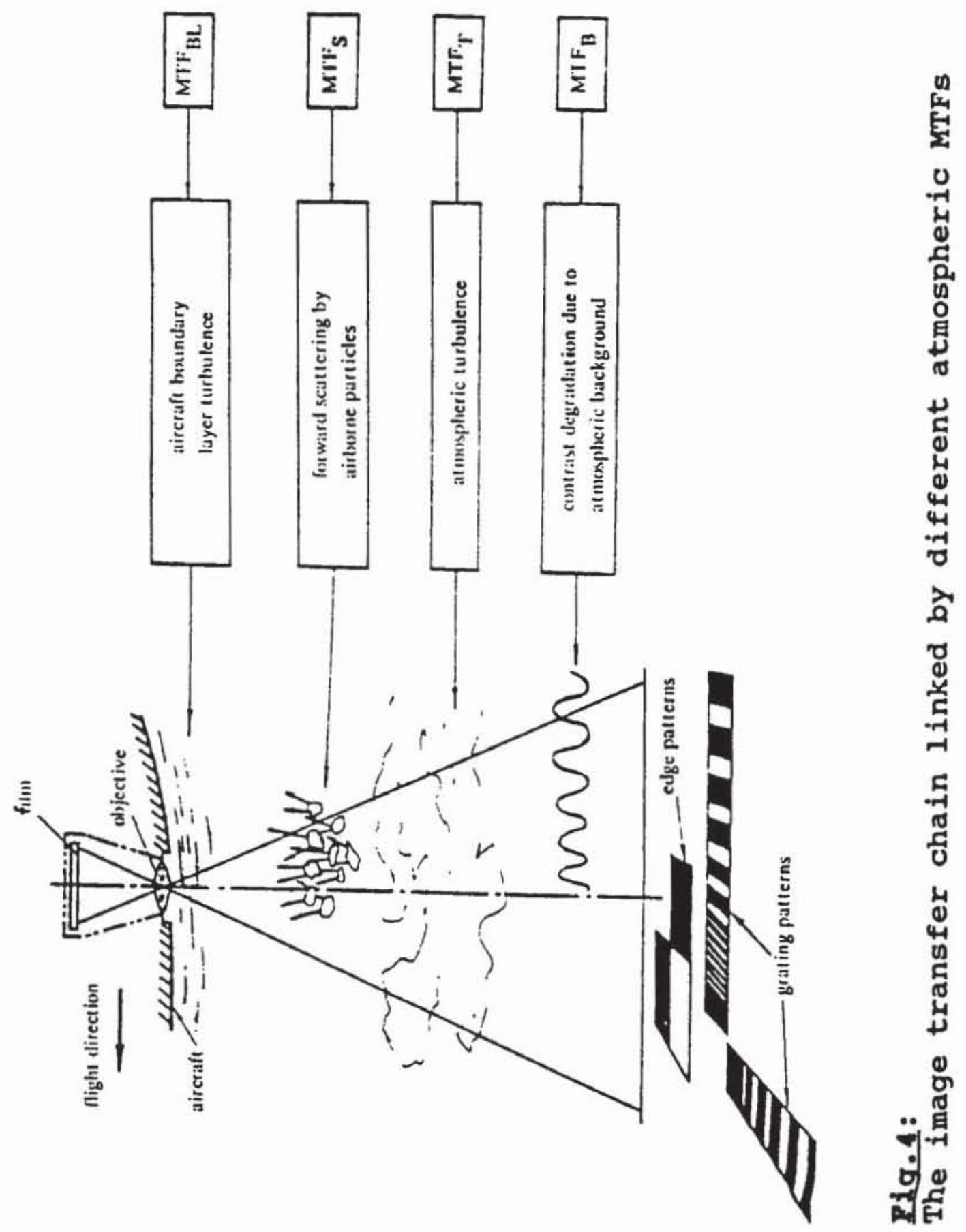



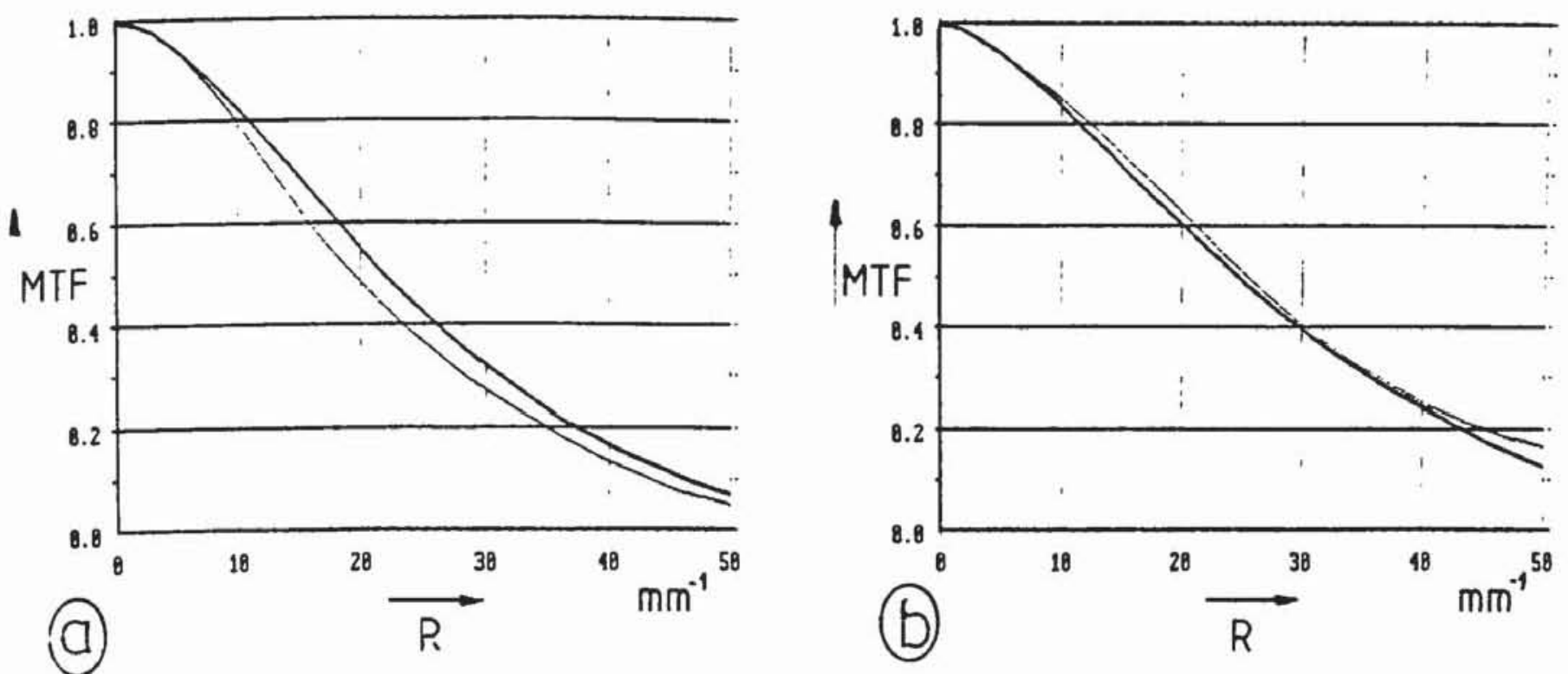

rig.5:

Airborne photographs taken at three different flight altitudes and the corresponding MTF curves
a) $\mathrm{H}=900 \mathrm{~m}$ above sea level
b) $\mathrm{H}=1920 \mathrm{~m}$ above sea level
c) $\mathrm{H}=3435 \mathrm{~m}$ above sea level

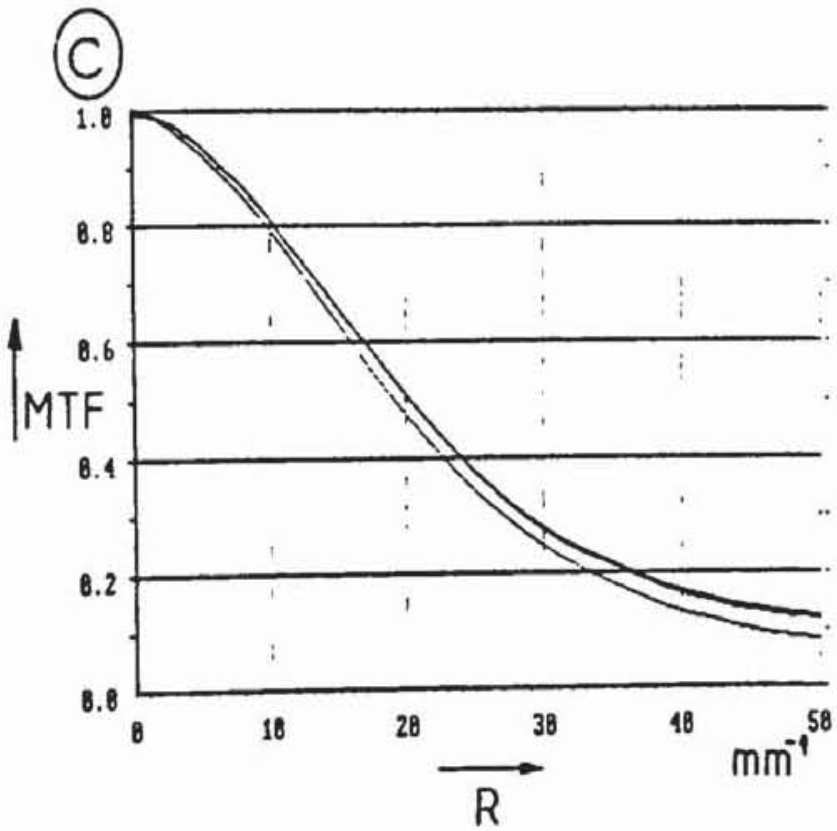

: MTF obtained with the edge image in $\mathrm{X}$-direction : MTF obtained with the edge image in $\mathrm{Y}$-direction 